\title{
Diverse Morphologies of Influenza Filament Budding: an Ultrastructural Study
}

\author{
Swetha Vijayakrishnan, Colin Loney and David Bhella \\ MRC-University of Glasgow Centre for Virus Research (CVR), 8 Church Street, Glasgow, UK
}

Commonly known as the flu, Influenza is caused by the single stranded RNA viruses of the Orthomyxoviridiae family. This virus causes seasonal epidemics every year and occasional pandemics infecting millions of people worldwide. Influenza viruses exhibit striking variations in morphology between strains and are characterized as being either filamentous or spherical. While the filamentous form has been attributed to clinical samples isolated from patients [1], virus cultivated in the laboratory are predominantly spherical [2]. The exact role of the filamentous form in viral infection and pathogenicity remains undetermined. It is thought that they may play an important role in cell-to-cell transmission of virus or facilitate release of smaller particles by disrupting the mucus layer in the respiratory tract, which is the primary target during Influenza infection. Therefore determining the structure of filamentous flu is crucial towards determining its function.

Here we report results of the first three-dimensional study of the ultrastructure of filamentous flu budding from infected cells using cryo electron microscopy (CEM), cryo electron tomography (CET) and confocal microscopy.

To obtain an overview of filament formation in flu-infected cells, we performed confocal imaging. An abundance of viral filaments were seen, with the predominant form being several microns in length $(>10 \mu \mathrm{m})$, typically connected to the cell at one end and frequently had bulbous heads at the other, and resembled Archetti bodies [3]. These studies unequivocally confirmed the position of the bulbous heads to be at the ends furthest from the cell (distal/leading ends, Fig. 1A).

CET of infected cells showed a profusion of viral filaments and Archetti bodies, all of which were densely covered in surface spikes and had a continuous membrane (Fig. 1B). The majority of filaments extended beyond $10 \mu \mathrm{m}$ in length and had a diameter of about $74 \mathrm{~nm}$, while diameters of the bulbous heads of Archetti bodies ranged from 200 to $550 \mathrm{~nm}$. While some Archetti bodies contained tubules of a scaffold-protein M1 that usually lines the inner surface of the viral membrane, none had any ribonucleoproteins (RNPs), and hence were devoid of any genetic content (Fig. 2A). Interestingly, the majority of long filaments that did not have bulbous heads were empty with only some seen to bear RNPs at their leading ends.

CET imaging of purified virions revealed two distinct classes of filaments; short capsule-shaped (that we term as bacilliform, diameter $95 \mathrm{~nm}$ ) and long filaments (diameter $79 \mathrm{~nm}$, Fig. 2B). Bacilliform virions appeared well ordered with the RNPs seen to lie parallel to the long axis of the particle, adopting the known ordered " $7+1$ " arrangement of genome segments $[2,4]$. Long filaments however were narrower and mostly appeared to lack this feature, but often contained fibrillar material along their entire length. The scaffolding matrix protein M1 layer appeared continuous in both filaments and bacilliform virions.

To conclude, our data represents the first high-resolution three-dimensional structural analysis of influenza virus filaments budding from the host cell under near native conditions. This work provides new insights into virus structure, morphology and infection mechanisms. We believe that the important structural differences between these diverse classes of particles imply there are two 
distinct pathways of morphogenesis for the small virions and long filaments. We also hypothesize that the absence of genome segments in long filaments and Archetti bodies indicate a distinct role for these particles during the infectious cycle possibly to combat the immune response or help in the spread of the smaller virus particles.

\section{References:}

[1] BE Eddy and RW Wyckoff, Proc Soc Exp Biol Med 75 (1950), p. 290-293.

[2] A Harris et al, Proc Natl Acad Sci USA 103 (2006), p. 19123-19127.

[3] I Archetti, Archiv fur die gesamte Virusforschung 6 (1955), p. 29-35.

[4] T Noda et al, Nature 439 (2006), p. 490-492.

[5] The authors acknowledge funding support from the Medical Research Council (MRC), UK. Profs. Massimo Palmarini, John McLauchlan and Rick Randall are thanked for their useful discussions and critical reading of the manuscript.

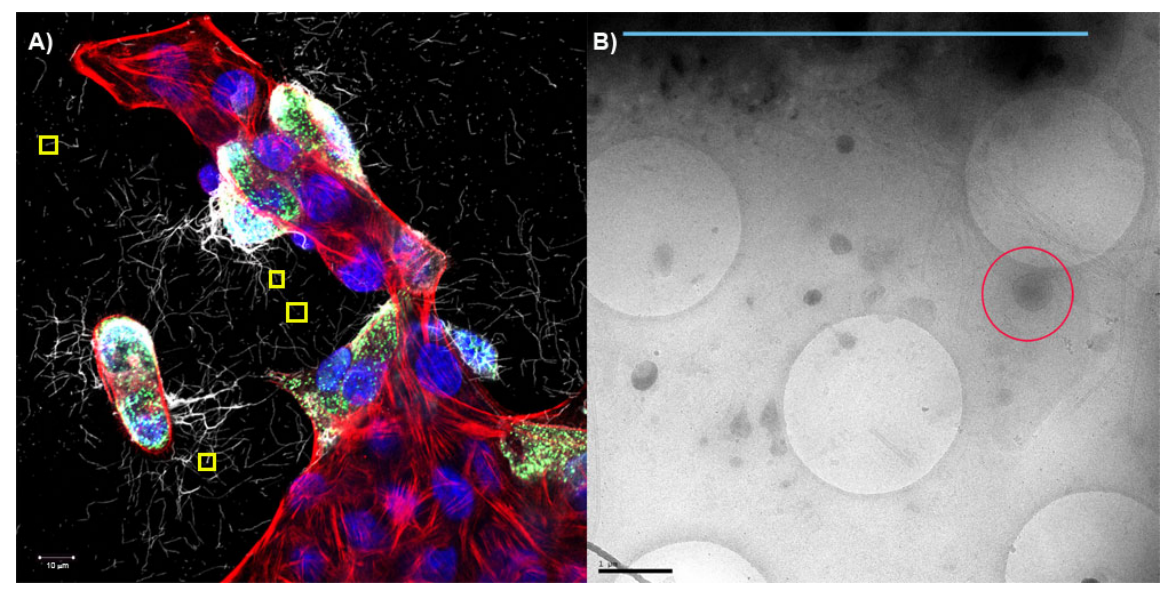

Figure 1. Budding of Influenza virus from infected cells revealed a profusion of filaments. (A) Confocal image revealed filaments greater than $10 \mu \mathrm{m}$ with several having bulbous heads (yellow square) at the distal/leading ends. These structures are termed Archetti bodies. (B) Cryo EM shows several filaments budding from the cell edge (blue line) including Archetti bodies (red circle) that seem devoid of any genomic content.

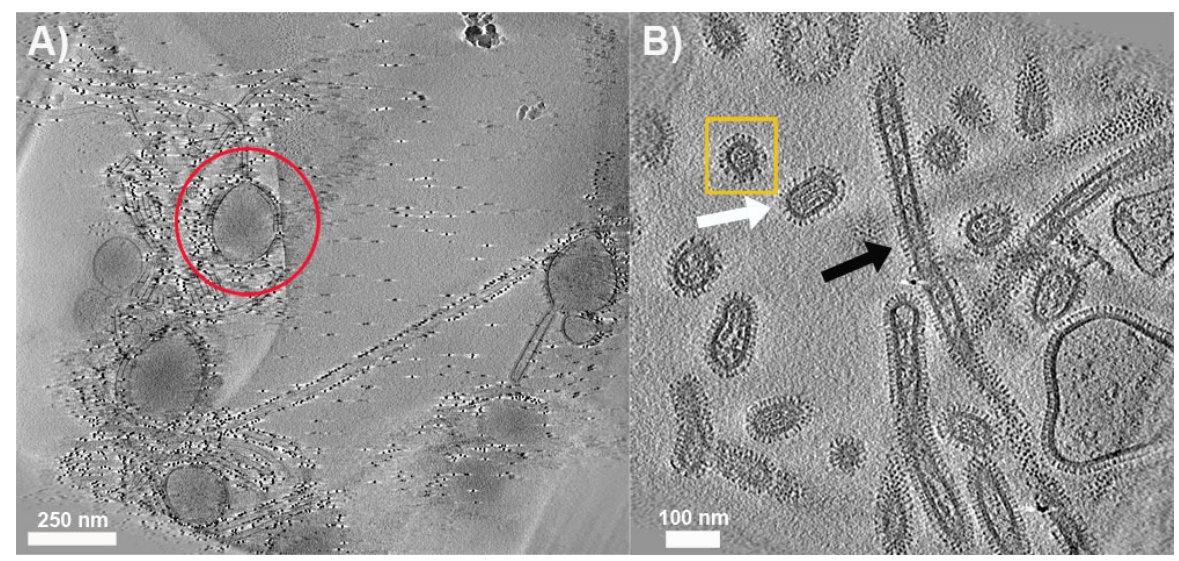

Figure 2. CET of Influenza filaments from cells and purified preparations show greater detail of structure and morphology. (A) While Archetti bodies (red circle) and straight filaments bud from cells, (B) purified virus show 2 major classes; bacilliform (white arrow) and long filaments (black arrow). The RNPs show a classic " $7+1$ " arrangement (yellow square) in the former while the latter seem to be devoid of recognisable genome segments. 